Review

\title{
Technologies and Applications Concomitant with Web-Based GIS
}

\section{Sadiq Mukhtar}

Department of Geography, Bayero University, Kano, Nigeria; smukhtar.geog@buk.edu.ng or abubsadiq@gmail.com

\begin{abstract}
Web-Based GIS is a platform that deals with the representation, management and storage of locational information for real-time online retrieval, viewing and manipulations. Web-based GIS incorporate all the functions of information systems: gathering, storing, retrieving, visualizing and dissemination of spatial information on the Internet. In modern-day GIS serve as vital means to not only geo-referenced locational information that are been retrieved but also the development in web-based technologies as well as there involvements in decision making processes. In recent time the increased used of GIS on the Internet have totally transformed and made three distinct changes across our GIS environment. These are: (i) how are GIS data being accessed (ii) what are modes of its transmission and (iii) what available GIS functionalities are within the environment for analysis. This paper examines the applications and technologies associated with webbased GIS architectures with an emphasis on the internet GIS based structure and case studies of GIS Web-based applications in MapInfo, Microsoft Silverlight Technology and the ArcGIS Server using ArcGIS JavaScript API.
\end{abstract}

Keywords: web-based GIS; application; architectures; technology

\section{Introduction}

Web based GIS serves as a platform that deals with the representation, management, as well as a storehouse of location information that uses the adoption of both wired and wireless connection to retrieve important information on the web or online (Peng and Tsou 2003; Tsou,2004). The modern day GIS applications steadily serve as very vital channels to not only geo-referenced information that are been retrieved by the public, but also the 
evolution in web technology as well as the involvement in official decision making processes (Sakamoto and Hiromichi, 2004). In today's world the internet has totally transformed and made three distinct changes across our GIS environment. These are: (i) how are GIS data being accessed (ii) its mode of transmission and (iii) the availability of GIS analysis functionalities within its environs (Peng n.d.). Accessing these various forms of GIS functionality from the web browsers doesn't necessarily mean they require buying proprietary GIS software.

\section{Web Mapping Technology Visualisation}

Visualisation is a basic key to how geographic data can be represented. Each website has its own type of mapping application it utilises for its web based GIS application (Adnan et al 2010). Steady improvements in usability through improved user interfaces also account for the increased popularity of visualization techniques (Aoidh et al 2008). There are three common techniques web based GIS mapping solution visualization adhere to namely:

Static Map Renderers: this method stands out because of its rendering functionality which is quite fast but has lapses with its flexibility thereby leading to a non-interactive phenomenon.

Slippy (Tile based) Maps: It is fast, dynamic and an extensively used geo-visualisation technique. It works such that the map can be divided into separate numbers of zoom levels leading to each zoom level having an identical number of tiles. A number of popular web based GIS mapping sites use this technique namely: Google Maps, Microsoft Virtual Earth, Yahoo Bing Maps, open layers etc.

Flash Mapping: A very vital issue that can even be unified as an API into ArcGIS which is known as the ArcGIS Flex API. 


\section{Overview of Web Based GIS Architectures}

Web GIS technology enables the addition of several GIS functionality which also involve the integration of data from disparate sources that are been found on a wide range of computer application that are located within a network. Several types of architectures can be used in the development of web-based GIS application (Adnan et al, 2010). They include (i) 2-tier architectures and (ii) 3-tier architecture. The 2-tier architecture help preserve the user's interface and vital functional part of the web application on the first tier and transfers the database and the functions that aid in data storage on the second tier while 3-tier architecture have both their interface and data storage functions dispersed differently on different tiers such that changes effected don't affect the entire web environment.

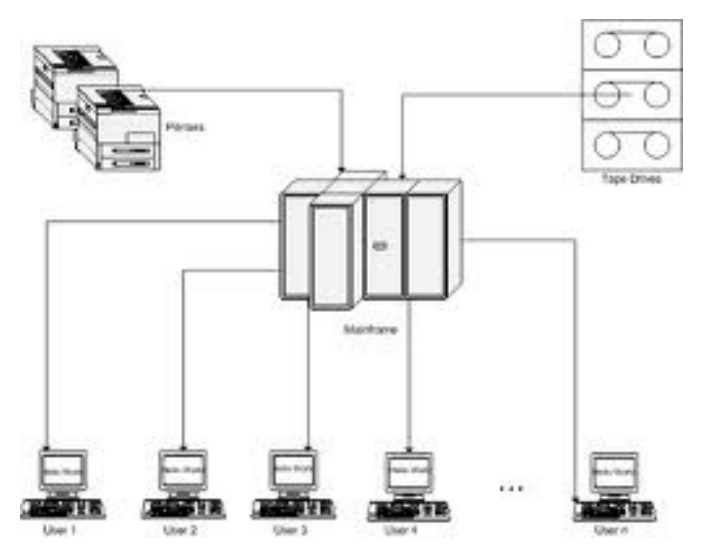

Fig 1: showing a 1or n-tier architecture 


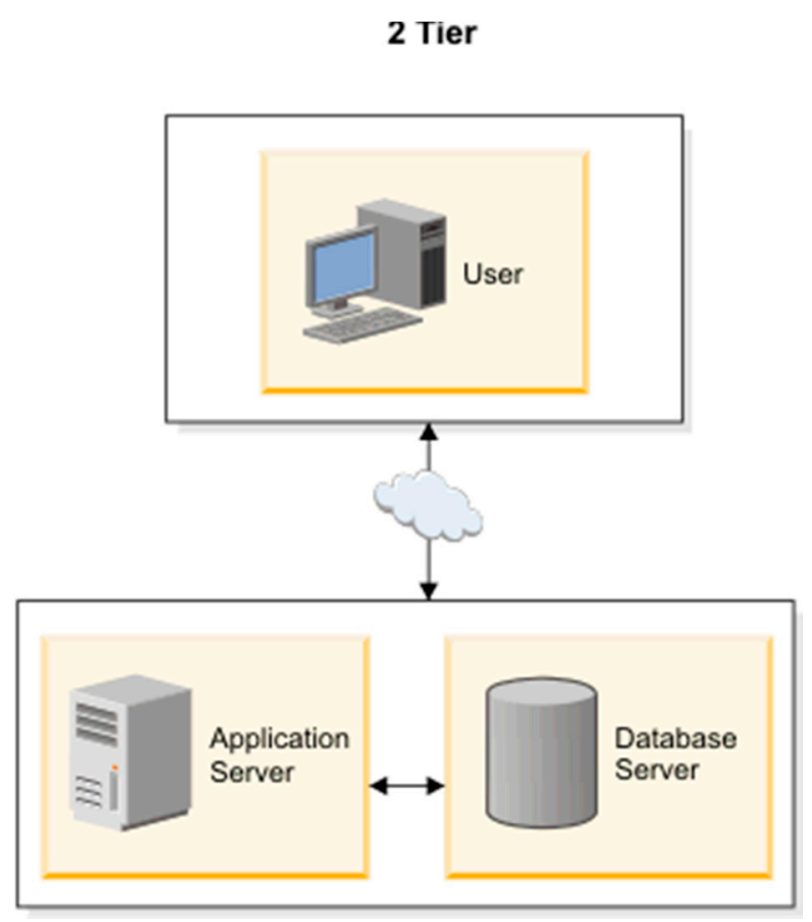

Fig 2: showing a 2-tier architecture

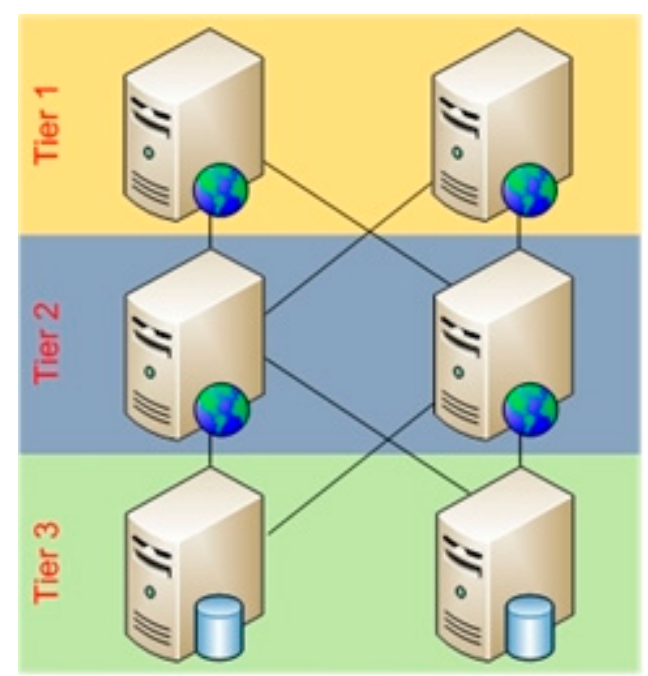

Fig 3: showing a 3-tier web based GIS application architecture

The figure above indicate a stepwise or workflow of a 3-tier architecture which a web based GIS application must adhere to. This architecture is such that the $1^{\text {st }}$ tier stands or represents the user interface, the $2^{\text {nd }}$ tier denotes the business logic functions which can also be referred to as the functional part of the web application. A mapping service is embedded in 
this functional section which further generates and brings out an array of map(s) to the users.

Furthermore, the file service writes necessary data into XML files while interactions between remote applications to acquire live feeds of data as specified is bent on the communication service function.

\section{Internet GIS Structure}

\section{Server Side Application}

The server processes the request and sends back the results to the user client. Data demanded by the customer and corresponding processing tools are gotten from the server. Its mode of operation works such that the application uses internet communication software i.e Microsoft(NetMeeting) which have the presence of simple HTML to Common Gateway Interfaces approach and as well as the application approach (Peng n.d.). The growth of development of was as a result of GIS software developers that helped in the origination of this Common Gateway Interface wrappers to stand alone GIS that made the availability and provision of map visualisation and simple map query capabilities (Vermes, 2006). A noticeable drawback found is the interaction between the internet user and the GIS analysis because basically the GIS executes the requested analysis, while the graphics and text output are readily available to the web server via the GIS. In turn the web server further delivers the processed output to the internet end users. 


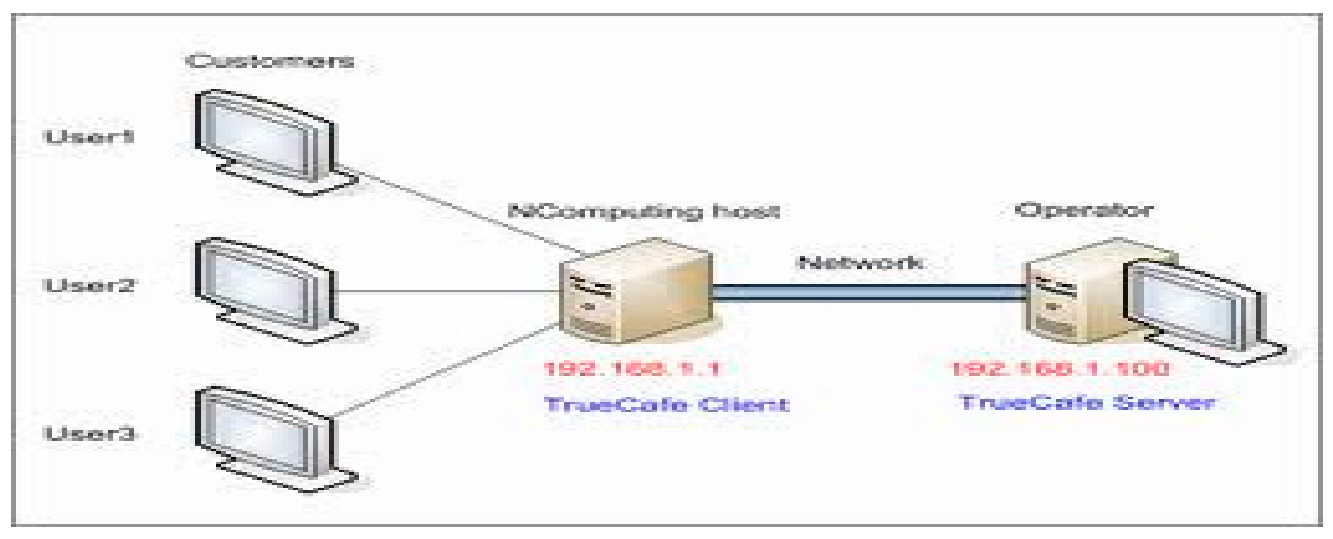

Fig 4: a typical server side workflow operation

\section{Client Side}

With the advancement in technology client side GIS has been a more preferred focal point for web GIS technologies because it offers more programming plug-ins such as java applets, Active $\mathrm{X}$ controls and extendable web clients which help extend the capabilities of the web browsers (Zhao et. al, n.d.). This is primarily achieved in such a way that GIS analysis and data to be processed are done via the web browser in the user's desktop/local machine. This plugs in acts as GIS data translators and filters which are infused within the HTML document which appear more like the embedded giff or jpeg map image. They enable spatial data interaction and manipulation within its interface. The server retains the GIS data and the corresponding analytical tools and sent on request from the server to the client which eventually handles the local processing of the GIS data. Some lapses with this GIS plug-ins is that they are platform neutral in the sense that specific plug-ins must be created or tailored specifically for a respective operating system. Secondly it has to be installed on the primary memory if the end users machine which could further occupy space. 


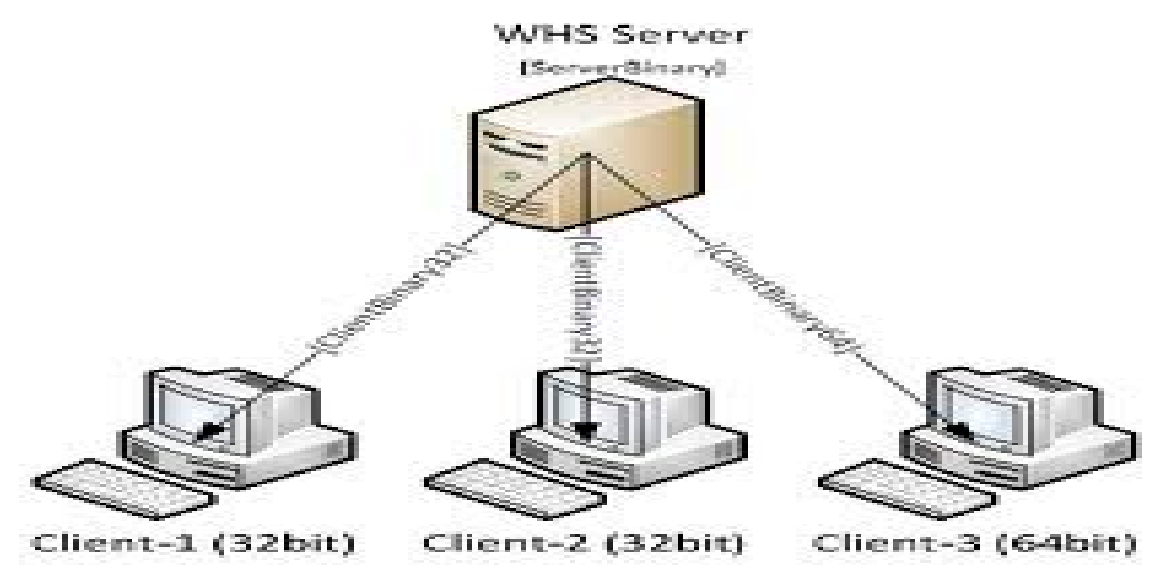

Fig 5: showing a client side interaction

\section{Case Studies}

Commercial Technology: MapXtreme (MapInfo)

MapXtreme (MapInfo): it's a Java based web mapping technology which enables developers to stream vector based data down to an applet running into the clients browser, the clients can work within the browser on their machines and only communicate with the server when a new request is been modified or stored up (Hogan, n.d.). It is such that the client accesses a browser to request a dynamic map which is transferred onto the web server. The webserver is responsible for geocoding the request of a dynamic map page and sends it across to the application server which is the engine room that permits the operations of the mapping solution which handles the requested operation of the client. The specified requests are sent to the map server in which MapX is embedded. A Giff like image or JPEG is deduced from the Map server which is then transported to the application server to be unified into an HTML page which eventually leads to the clients end result.

\section{Coles supermarket store locator:}

http://www.coles.com.au/Stores-Services/Locations-trading-hours.aspx 
Though the map seems static, it supports an interactive phenomenon which allows you to be able to pan, zoom in and out. It utilises MapInfo MapXtreme which exist as both internet and intranet packages. It performs such queries as location specifications i.e. "finding the nearest applications executed by a geocoding engine. Spatial queries involving distributions can also be performed to discover readily available services that are belong to the client specified address. The end result information is a highlighted in the map indicating the users address, its relative close services and details as regards other concerning services.

\section{Microsoft Silverlight technology: http://maps.bouldercounty.org/boco/emapping/}

The architecture involved adheres to the Client side interaction: here the web server is at the receiving end from the specified tasks and conveys the appropriate HTML file. It is the responsibility of the web server to decode the html file and locate the java applet tags which are embedded in the web GIs server. This site is primarily targeted at both road location directions i.e. minor highways and local roads are shown when zooming in and out as well as distinguishing between various property/parcels. Interaction is so much enhanced and supported in this web site. It has some form of rendering functionalities that allows both street viewing and some sort of digital elevation models, advanced tools are also present within this site that give the user ability to manipulate functions like latitude/longitude, buffer and measure formats etc. Firstly, the user enters a request by typing the address or URL into the web browser that is been sent to the web server which further locates the HTML tag and the resultant effect is brought out on display. Various input queries can be performed that are as well been sent to the web server using the Silverlight applications that searches for the corresponding html tags that are fused into the JavaScript. The characteristics of this JavaScript and html tags are so linked such that special programs are responsible for each query that is been performed which reside in the web GIS server that 
now interprets and manipulates the data which is then sent through the web browser based on the input query by the user.

\section{ArcGIS Server using ArcGIS JavaScript API}

\section{Flagler county property Appraiser:}

http://gis.fltplan.com/tracking/index.html?tailnumber=N852AL\&username=re\&zoomto=1298BC4rt $\underline{\text { \&remotecode }=6487 \mathrm{P}}$

This site complies with the client side behavior. It uses ArcGIS JavaScript API and capabilities which is solely dependent on an ArcGIS server and cold fusion 8Ajax capabilities which helps to infuse GIS maps and task in a web package. The presence of these cold fusion helps in the connection of the html files into a database. The JavaScript code is found inside the html page and this application helps to access information from diverse servers in one application. It supports different projection and visualization of map services from ArcGIS server and also important GIS querying such as finding locations and geo-processing are operational (ESRI, n.d.). This website works as an application such that real property information and taxable values can be discovered by seeking end users.

\section{Conclusion}

Web based GIS has become a sort after necessity in our environment and will continue to be as a result of its giant strides from ordinary maps to dynamic maps that are been fused into the internet. Therefore, its role is such that it has come to stay and will continue to evolve in trying to reshape our daily lives.

\section{Acknowledgement}

This work was submitted as part of my coursework for the course Principles of WEB-Based GIS to the University of South Wales formerly University of Glamorgan for the requirement for the award of Msc Geographical Information System.

\section{References}


Adnan, M., Singleton, A.D., and Longley, P.A. (2010) Developing Efficient Web-Based GIS Applications. Available from: [online].

http://discovery.ucl.ac.uk/19247/1/19247.pdf Retrieved on 19 Feb. 2011.

Aoidh, E. M., Bertolotto, Wilson, D.C. (2008). "Understand geospatial interests visualising map interaction behaviour". GeoVisualization of Dynamics, Movement and Change, $\quad 7(3), 275-286$.

C. L. Hogan n.d., Mapping on the web using mapinfo products. Available from [online]: http://www.gisdevelopment.net/technology/gis/techgi0022.htm Retrieved on 19 Feb.

2011

ESRI n.d., Available from: [online] http://resources.esri.com/help/9.3/arcgisserver/apis/javascript/arcgis/help/jshelp star t. $\mathrm{htm} \#$ jshelp/overview api.htm Retrieved on $20^{\text {th }}$ March 2011

Liu Yong, Li Qing Quan, Xie ZhiYing, Wang Chong (n.d.) Research of Mobile GIS Application Based on Hand Held Computer. Available from: [online]. http://www.isprs.org/proceedings/XXXIV/part4/pdfpapers/475.pdf Retrieved on $20 \mathrm{Feb}$.

2011

Peng, Z. R. and Tsou, M. (2003) Internet GIS: Distributed Geographic Information Services for the Internet and Wireless Networks John Wiles and Sons Inc. Hoboken, NJ, USA. p. xxv Available from: [online]. http://www.ejisdc.org/ojs2/index.php/ejisdc/article/viewFile/584/292 Retrieved on 20 Feb.2011

Sakamoto, I. and Hiromichi, F. (2004) Development and Application of a Livable Environment Evaluation Support System Using Web GIS. Journal of Geographical Systems, 6, 175-195.Available from:[online]. http://www.ejisdc.org/ojs2/index.php/ejisdc/article/viewFile/584/292 Retrieved on

Vermes, J. (2006). Is Web Technology Disrupting Traditional GIS? Geospatial Solutions, September, 13-15, Available from: [online] http://www.geospatialsolutions.com/geospatialsolutions/Net+Results/Is-Web-Technology-DisruptingTraditional-GIS/ArticleStandard/Article/detail/373360 . Retrieved on 19 Feb. 2011.

Zhao, F.J., Zhang, J.F. \& Cao, D.Y. (n.d.) Dynamic Database Connection and Dynamic Web Map Service for Internet Mapping [online]. Available from: http://ieeexplore.ieee.org/stamp/stamp.jsp?tp=\&arnumber=1526512\&isnumbe $\underline{\mathrm{r}} \quad=32599$ Retrieved on $19^{\text {th }}$ February 2011. 